\title{
Super-adsorbent material based on functional polymer particles with a multilevel porous structure
}

\author{
Dong Chen ${ }^{1,2}$, Li Wang ${ }^{1}$, Yuhong $\mathrm{Ma}^{2}$ and Wantai Yang ${ }^{1,2}$ \\ Porous polymer particles with multilevel structures consisting of submicron-sized hollow cavities and interconnected mesopores \\ within the crosslinked shell were designed and prepared. An extremely high density of anhydride groups in the crosslinked shell \\ can be conveniently converted into versatile functional groups for the adsorption of hazardous pollutants. As a proof of concept, \\ we utilized carboxylate-functionalized hollow particles as a selective adsorbent for the removal of the basic dye methylene blue \\ and found that these particles exhibited a very high adsorption capacity $\left(1603 \mathrm{mg} \mathrm{g}^{-1}\right)$. Furthermore, the hollow structure, highly \\ interconnected mesopores and rigid shell of the particles not only endow the adsorbent with fast adsorption/desorption rates but \\ also facilitate their facile separation and easy regeneration. We believe that these porous polymer particles have great potential \\ for applications in many fields, including as adsorbents, catalyst supports and matrices for the binding of sensitive materials. \\ NPG Asia Materials (2016) 8, e301; doi:10.1038/am.2016.117; published online 26 August 2016
}

\section{INTRODUCTION}

Adsorbent materials are of great scientific and technological interest owing to their ability to interact with specific substances and efficiently separate them from a mixture. ${ }^{1}$ Owing to their superior physical and chemical features, including a large specific surface area, unique pore structure and tunable pore surface property, adsorbent materials have found utility in multiple applications, such as separation/purification, ${ }^{2}$ as catalyst supports, ${ }^{3}$ adsorption, ${ }^{4}$ drug delivery, ${ }^{5}$ gas separation/ storage $^{6}$ electrode materials for electrochemical double layer capacitors and fuel cells ${ }^{7,8}$ and so on.

To meet the demand for green chemistry and sustainable development, tremendous endeavors have been devoted to the design and synthesis of advanced adsorbent materials, which is crucial for efficient adsorption, separation and purification. Generally, an effective adsorbent should be a solid material with high mechanical and chemical stability, which makes it suitable for application under severe conditions, that is, easily separated, regenerated and reused. Second, a good adsorbent material should possess a proper porous structure, endowing it with a large contact area and facilitating mass diffusion within the porous media. Third, the porous surface should contain a large number of functional groups, which helps to determine its adsorption selectivity and capacity. ${ }^{9-11}$ Owing to exponential growth in the field of nanomaterials over the last two decades, we have witnessed the rapid development of high-performance nanostructured adsorbents. Numerous nanomaterials with well-defined porosities and controllable surface properties have been designed, synthesized and used as adsorbents for desired applications, including for $\mathrm{CO}_{2}$ capture, ${ }^{12,13}$ for the separation and storage of fuel gas ${ }^{14}$ and especially for the elimination of pollutants in aqueous effluents. ${ }^{15,16}$

In recent years, water contamination has become a serious environmental problem, and a huge amount of toxic dyes have been discharged directly into the environment. Various technologies have been developed to solve this problem, such as adsorption, ${ }^{17}$ coagulation/flocculation, ${ }^{18}$ oxidation, ${ }^{19}$ photodegradation, ${ }^{20}$ membrane filtration ${ }^{21}$ and the harnessing of biological processes. ${ }^{22}$ Pollutant remediation via adsorption processes is considered to be one of the most promising techniques owing to operation simplicity, high efficiency and applicability for the treatment of wastewater on a large scale. ${ }^{23}$ In addition to the traditional activated carbon, ${ }^{24}$ different types of adsorbent materials have been developed, such as natural or synthetic zeolites, ${ }^{25}$ organically modified porous silica, ${ }^{26}$ natural or modified clays ${ }^{27}$ and macroporous polymeric adsorbents. ${ }^{28}$ However, inherent drawbacks for such materials include high operation costs, long time consumption, low selectivity, limited adsorption capacities and difficulties in their regeneration and reuse. Therefore, it is of great theoretical and practical significance to develop high-performance, low-cost and recyclable adsorbents.

Currently, research interest in adsorbent development is mainly concentrated on the functionality of the pore surface and the design of pore size and geometry. 9,11 To endow the porous materials with high selectivity and adsorption capacity, a high density of functional groups should be grafted onto the pore surface. ${ }^{29,30}$ Mesoporous silica functionalized with a high density of carboxylate groups can serve as an efficient adsorbent for the selective removal of basic dyes. ${ }^{26}$ In addition to the content of functional groups, the appropriate pore

\footnotetext{
${ }^{1}$ State Key Laboratory of Chemical Resource Engineering, Beijing University of Chemical Technology, Beijing, China and ${ }^{2}$ Key Laboratory of Carbon Fiber and Functional Polymers of the Ministry of Education, School of Materials Science and Engineering, Beijing University of Chemical Technology, Beijing, China

Correspondence: Professor W Yang, State Key Laboratory of Chemical Resource Engineering, Beijing University of Chemical Technology, No. 15 North Third Ring Road East Road, Chaoyang District, Beijing 100029, China.

E-mail: yangwt@mail.buct.edu.cn

Received 10 March 2016; revised 9 June 2016; accepted 14 June 2016
} 
a

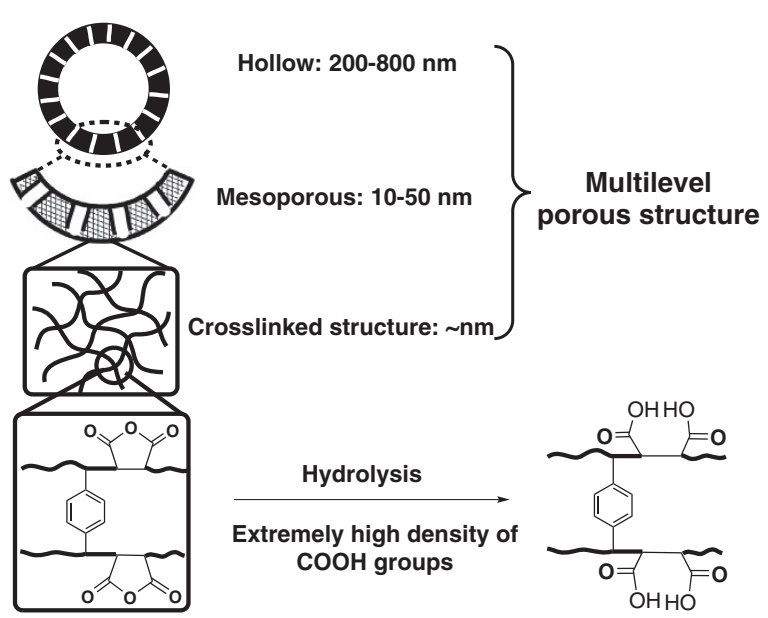

b

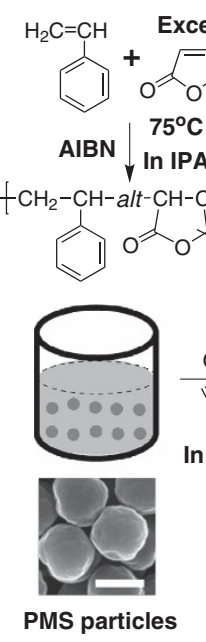

DVB-MAH crosslinked shell

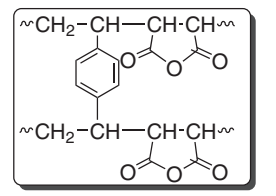

Figure 1 Structure of the as-designed multilevel structured porous MSPP (a) and synthetic route (b). Scale bar: $1 \mu$ m.

structure also has a crucial role. The pore structure of conventional adsorbents is mainly composed of micropores, which are not applicable for dye molecules with large size. To overcome the disadvantage of micropores, adsorbents with mesopores or even hierarchically porous structures have been designed (for example, bimodal macrostructure-mesostructure and multilevel porous structures). ${ }^{31,32}$ The micropores and mesopores provide the adsorbent with a large specific surface area combined with a high density of functional groups, leading to a high adsorption capacity. Meanwhile, the presence of macropores and interconnected mesopores enables the fast diffusion of molecules into the internal pores (especially for large molecules), which greatly improves the adsorption rate. ${ }^{33-35}$ However, the tedious preparation procedures for the hierarchically porous adsorbents greatly hinder their practical applications. Furthermore, there are still some inaccessible binding sites in such materials, leading to binding efficiencies far below $100 \%$ (relative to the molar amount of the functional groups).

In recent years, functional hydrogels have emerged as effective adsorbents for the removal of water-soluble contaminants. Compared with conventional adsorbents having porous structures, hydrogels present several advantages. For instance, the 3D network of the hydrogel is formed through the physical/chemical crosslinking reactions of various monomers, and the precise control of functionality can be achieved through structure design at a molecular level. The functional groups in the $3 \mathrm{D}$ network can equivalently conjugate with target molecules, leading to unique selectivity and remarkable adsorption capacity. ${ }^{36-38}$ However, for amorphous soft materials such as hydrogels, a relatively long time is required to reach a saturated state of adsorption. Meanwhile, difficulties associated with the regeneration and disposal of hydrogel adsorbents constitute major obstacles for their practical application.

Based on the above discussion with an aim to combine the advantages of traditional porous adsorbents and those of polymer gels while simultaneously overcoming their disadvantages, we designed and synthesized a novel type of porous particle with a unique multilevel structure. As shown in Figure 1a, the as-designed multilevel structured porous polymer particle (MSPP) has three levels of porous structures: (1) hollow voids obtained from the removal of template cores, (2) interconnected mesopores in the shell, and (3) 3D networks formed by the crosslinked alternating copolymerization of maleic anhydride (MAH) and divinyl benzene (DVB).
An extremely high density of reactive anhydride groups in the crosslinked shell could be conveniently converted into other versatile functional groups, such as $\mathrm{COOH}$ or $\mathrm{NH}_{2}$. When used as adsorbent in aqueous solution, the $3 \mathrm{D}$ networks could be swollen to some extent. Consequently, the functional groups in the $3 \mathrm{D}$ networks could equivalently conjugate with the targeted molecules, leading to a remarkable adsorption capacity. Additionally, the hollow voids and mesoporous channels provide a high contact area and fast diffusion path for the adsorption and subsequent desorption of the targeted molecules, especially for dyes with a high molecular weight and large size. Furthermore, the highly crosslinked shell has excellent mechanical strength, which is beneficial for the separation, regeneration and reuse of the MSPPs. These MSPPs integrate the advantages of both porous materials and functional polymer gels and show great potential for practical applications in pollutant removal.

As described in Figure 1b, the MSPPs were synthesized using a one-pot method. This process is an improvement over our previously developed 'stabilizer-free precipitation polymerization' for core-shell polymer particles and consequent removal of the template core by solvent etching. ${ }^{39,40}$ Excess MAH was added into the reaction system at the very beginning of the reaction. After the alternating copolymerization of MAH and styrene (St), residual MAH monomer is present along with the monodisperse poly(MAH-alt-St) (PMS) nanoparticles formed in the reaction system. DVB was then added and copolymerized with the residual MAH to form the crosslinked shell. It is worth noting that the molar amount of the vinyl groups of DVB was equal to that of the residual $\mathrm{MAH}$, resulting in a highly crosslinked structure with a mesoporous shell layer. The PMS cores could be readily removed by solvent dissolution, and MSPPs were successfully obtained.

This facile one-pot approach for the preparation of MSPPs is advantageous in several aspects compared with conventional approaches. First, the PMS template and the DVB-MAH crosslinked shell are formed through precipitation polymerization without the use of any stabilizer. As a result, the product is impurity free and can be used directly without further purification. Second, the fabrication of MSPPs is a facile one-pot process with remarkably high yield and high efficiency (monomer conversion is nearly 100\%, and solid content can reach up to $30 \mathrm{wt} \%$ ), and the process is easy to scale up. More importantly, there is an extremely high density of functional groups in the rigid crosslinked shell, which is essential for its application as an 
advanced adsorbent. Third, the particle size and shell thickness of the MSPPs can be easily controlled by adjusting the monomer concentrations. Notably, the porous structure of the MSPPs can be adjusted by varying the composition of the solvent mixture.

\section{EXPERIMENTAL PROCEDURES}

\section{Preparation of the PMS template}

The preparation of PMS template particles was similar to that of poly(maleic anhydride-alt-vinyl acetate) particles. ${ }^{39,40}$ In a typical experiment, the monomers MAH $(2.45 \mathrm{~g}, 25 \mathrm{mmol})$ and St $(1.3 \mathrm{~g}, 12.5 \mathrm{mmol})$, the initiator azobisisobutyronitrile (AIBN; $37.5 \mathrm{mg}, 1 \mathrm{wt} \%$ relative to the monomers) and the solvent isopentyl acetate $(25 \mathrm{ml})$ were added to a $100 \mathrm{ml}$ three-necked flask.
After all the reagents were dissolved, the solution was purged with $\mathrm{N}_{2}$ for $30 \mathrm{~min}$, and the flask was then placed in a water bath at $75^{\circ} \mathrm{C}$ for $1.5 \mathrm{~h}$. Monodispersed PMS particles sized $\sim 580 \mathrm{~nm}$ were prepared and used directly as the core template for the preparation of core-shell and hollow polymer particles.

\section{Preparation of core-shell and hollow polymer particles}

To the PMS particle dispersion ( 10.0 wt\% PMS, $30 \mathrm{ml}), n$-heptane $(12.5 \mathrm{ml})$, DVB ( $1.10 \mathrm{~g}, 13.0 \mathrm{mmol}$ in vinyl groups) and AIBN (0.005 g) were added, and the mixture was then kept at $75^{\circ} \mathrm{C}$ for another $3 \mathrm{~h}$ with stirring at a speed of 100 r.p.m. Core-shell particles with an average diameter of $760 \mathrm{~nm}$ were obtained. The suspension of core-shell particles was stirred for another $30 \mathrm{~min}$

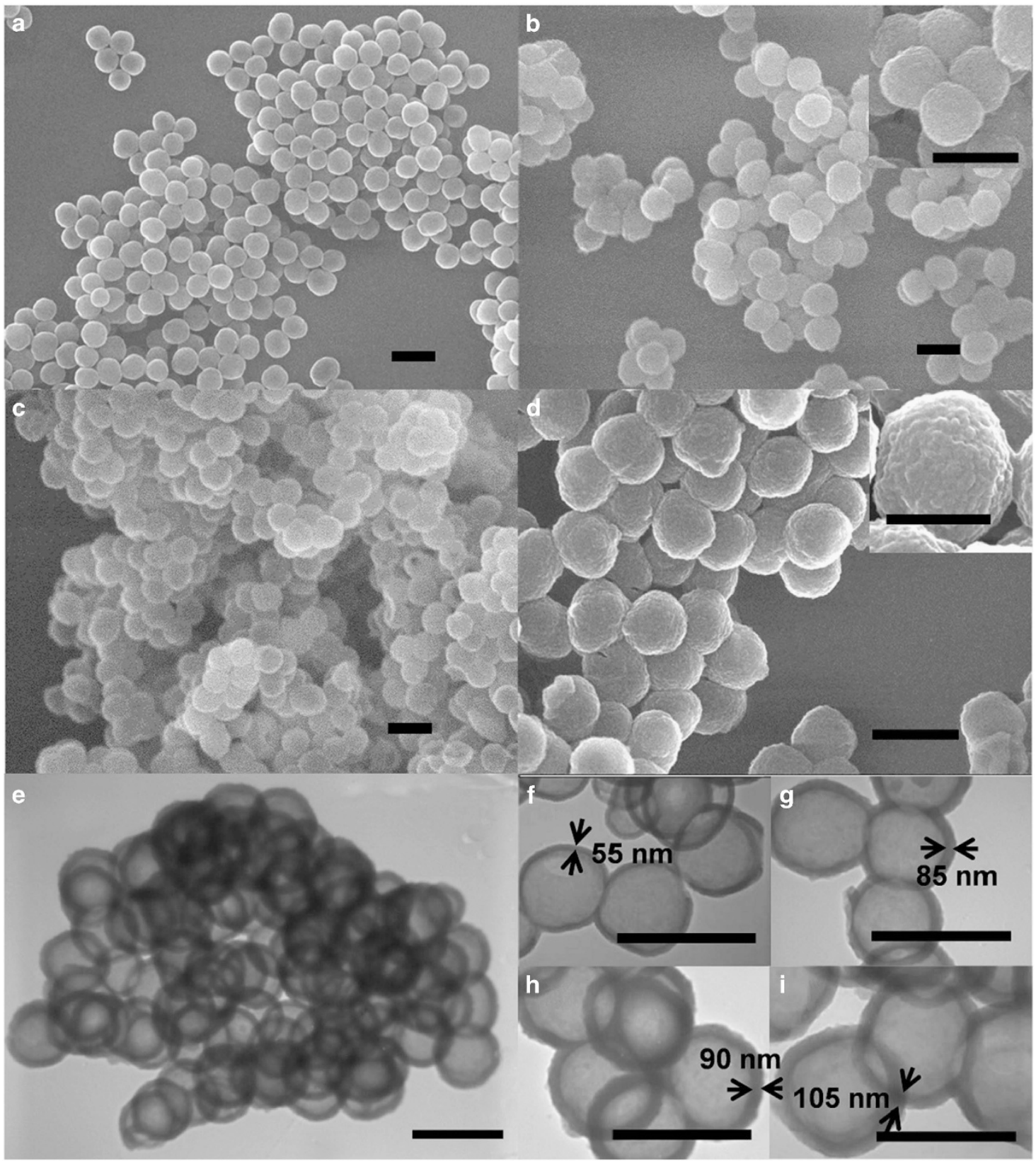

Figure 2 SEM images of (a) the PMS template; (b) the core-shell particles with DVB-MAH crosslinked shell; (c) the anhydride-functionalized MSPP-A; and (d) the hydrolyzed MSPP-A. (e) TEM image of the anhydride-functionalized MSPP-A. Reaction conditions for PMS synthesis: MAH $2.45 \mathrm{~g}$, St $1.3 \mathrm{~g}$, IPA $25 \mathrm{ml}$, AIBN $0.0375 \mathrm{~g}$, reaction temperature of $75^{\circ} \mathrm{C}$ for $1.5 \mathrm{~h}$. DVB $1.10 \mathrm{~g}$ and HP $12.5 \mathrm{ml}$ were then added to form the core-shell particles. (f-i) TEM images of the MSPPs with different initial DVB concentrations. The concentration of vinyl groups of DVB are $0.15,0.225,0.30$ and $0.375 \mathrm{~m}$, respectively. Scale bar: $1 \mu \mathrm{m}$, except (d, inset) $500 \mathrm{~nm}$. 
with the addition of acetone $(30 \mathrm{ml})$, then centrifuged and washed with acetone $(10 \mathrm{ml} \times 3)$ to remove the PMS template cores and to afford the anhydridefunctionalized MSPPs, which was designated as MSPP-A. MSPPs with different shell thickness were obtained by varying the DVB concentration. When the concentration of vinyl groups of DVB was $0.225,0.30$ and $0.375 \mathrm{M}$, MSPP-B, MSPP-A and MSPP-C were obtained, respectively. MSPPs with different mesoporous structures were obtained by changing the addition sequence of $n$-heptane (or adding different amounts of $n$-heptane). When $12.5 \mathrm{ml}$ $n$-heptane was added with isopentyl acetate during the preparation of the PMS template, MSPP-D was obtained. MSPP-E was obtained when $25 \mathrm{ml}$ of $n$-heptane was added during the shell formation process. Following reaction with $\mathrm{H}_{2} \mathrm{O}$ or $\mathrm{NaOH}$, the carboxylic acid and carboxylate-functionalized MSPPs were finally obtained.

\section{Preparation of non-hollow mesopore-free carboxylate- functionalized nanoparticles}

In a typical experiment, MAH $(1.225 \mathrm{~g}, 12.5 \mathrm{~mol})$ and DVB $(1.15 \mathrm{~g}, 13.0 \mathrm{mmol}$ in vinyl groups), AIBN (37.5 mg, $1 \mathrm{wt} \%$ of the monomers), isopentyl acetate $(25 \mathrm{ml})$ and $n$-heptane $(12.5 \mathrm{ml})$ were charged into a $100-\mathrm{ml}$ three-necked flask. After all the reagents were dissolved, the solution was purged with $\mathrm{N}_{2}$ for $30 \mathrm{~min}$, and the flask was then placed in a water bath at $75^{\circ} \mathrm{C}$ for $4.0 \mathrm{~h}$. The DVB-MAH particles, sized $\sim 320 \mathrm{~nm}$, were hydrolyzed with $\mathrm{NaOH}$ to obtain the carboxylate-functionalized nanoparticles.

\section{Characterization}

The morphology of the fabricated particles was observed using a field emission scanning electron microscopy (SEM; JSM-7600F, JEOL Ltd., Tokyo, Japan) and a transmission electron microscopy (TEM; H-800, Hitachi High-Technologies Corp., Tokyo, Japan). The chemical composition of the as-prepared particles was characterized by Fourier transform infrared (FT-IR) spectroscopy using a Thermo Nicolet Nexus 670 spectrometer (Thermo Nicolet Corp., Madison, WI, USA). The specific surface area and pore diameter of the hollow particles were determined by using a Quadrasorb SI analyzer (Quantachrome Instruments, Boynton Beach, FL, USA). The zeta potentials of the MSPPs were measured using a Brookhaven ZetaPALS zeta potential analyzer (Brookhaven Instruments Corp., Holtsville, NY, USA). The dye concentration in solution was measured using a UV-vis spectrophotometer and by measuring the absorbance at $663 \mathrm{~nm}$ (U-2910, Hitachi High-Technologies Corp., Tokyo, Japan).

\section{Dye adsorption study}

In a typical experiment, $10.0 \mathrm{mg}$ MSPPs and a $10 \mathrm{ml}$ aqueous solution of a given dye of a given concentration were placed in $50 \mathrm{ml}$ bottles and shaken in a

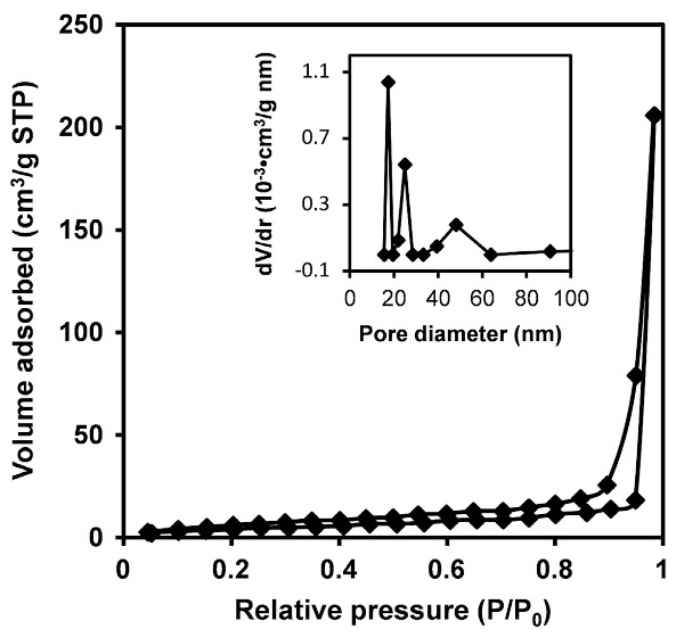

Figure $3 \mathrm{~N}_{2}$ isotherms of the hollow microspheres (MSPP-A) with a mesoporous shell at $77 \mathrm{~K}$. Inset: pore size distribution of the mesopores. thermostatic shaker at room temperature for $8 \mathrm{~h}$ to reach equilibrium. The $\mathrm{pH}$ of the solution was fixed at either 6 or 10 using dilute $\mathrm{HCl}$ or $\mathrm{NaOH}$ solutions. The bottles were then centrifuged at 4000 r.p.m. for 3 min to separate the absorbent from the system. The concentration of the dyes in the aqueous phase was determined by UV/vis spectroscopy by taking the maximum absorbance of each dye (663 and $513 \mathrm{~nm}$ for methylene blue (MB) and methyl blue, respectively).

\section{Desorption/reuse experiments}

To determine the reusability of the as-prepared MSPPs, adsorptiondesorption cycles were repeated 12 times with $\mathrm{MB}$, and the $\mathrm{pH}$ of the solution was fixed at 7. After dye adsorption, the dye-loaded MSPPs were placed in $10 \mathrm{ml}$ acidic ethanol $(\mathrm{pH}=2)$ and shaken at room temperature for desorption of basic MB (b-MB). After desorption in acidic ethanol 3-4 times, the regenerated MSPPs were washed and separated for the adsorption in the following cycle.

\section{RESULTS AND DISCUSSION}

Figures $2 \mathrm{a}$ and $\mathrm{b}$ show SEM images of the PMS template with an average diameter of $580 \mathrm{~nm}$ and core-shell particles with an average diameter of $760 \mathrm{~nm}$, respectively. The surface of the particles became relatively rough owing to the formation of the DVB-MAH crosslinked shell. Figures $2 \mathrm{c}$ and $\mathrm{d}$ are SEM images of the resulting MSPP-A before and after hydrolysis. Clearly, from Figure $2 \mathrm{c}$ the size and surface morphology of the particles remained unchanged after acetone extraction. Moreover, the shells of the particles remained intact even after being hydrolyzed, as shown in Figure $2 \mathrm{~d}$ and the inset, confirming the high stability and strength of the highly crosslinked shell. The TEM image of MSPP-A shown in Figure 2e clearly reveals that the PMS templates were thoroughly removed, and hollow spheres with a diameter of $760 \mathrm{~nm}$ and a shell thickness of $90 \mathrm{~nm}$ were successfully obtained. Additionally, the size of the MSPPs and the thickness of the crosslinked shell can be easily controlled by adjusting the concentration of DVB, with the shell thickness increasing from 55 to $105 \mathrm{~nm}$ with increasing DVB concentrations (as shown in Figures $2 \mathrm{f}-\mathrm{i}$ ).

Subsequently, the specific surface area and pore structure of the hydrolyzed MSPP-A were determined by $\mathrm{N}_{2}$ adsorption/desorption; Figure 3 shows the $\mathrm{N}_{2}$ isotherms at $77 \mathrm{~K}$. The $\mathrm{N}_{2}$ sorption results indicate the presence of mesopores in the crosslinked shell. Based on the Brunauer-Emmett-Teller and Barrett-Joyner-Halenda models, the specific surface area and the total pore volume of the MSPP-A were $21 \mathrm{~m}^{2} \mathrm{~g}^{-1}$ and $0.325 \mathrm{~cm}^{3} \mathrm{~g}^{-1}$, respectively. Additionally, the pore-size distribution was also calculated using the Barrett-JoynerHalenda method, and mesopores with diameters in the range of $17-48 \mathrm{~nm}$ were observed, as shown in the Figure 3 inset. These mesopores were formed owing to the contraction of the 3D networks resulting from the high degree of crosslinking during copolymerization of DVB and MAH.

The chemical structure of the anhydride, carboxylic acid and carboxylate-functionalized MSPPs was characterized by FT-IR spectroscopy. As shown in Supplementary Figure S1, the absorption bands at 1857 and $1780 \mathrm{~cm}^{-1}$, assigned to the $\mathrm{C}=\mathrm{O}$ stretching vibration of the anhydride groups, decreased and almost disappeared after the as-prepared MSPPs were hydrolyzed or neutralized with $\mathrm{NaOH}$. Meanwhile, new absorption bands appeared at 1728 and $1570 \mathrm{~cm}^{-1}$ owing to the formation of carboxylic acid and carboxylate groups, respectively.

The content of anhydride groups in the shell of the MSPP-A as determined by elemental analysis was approximately $52.8 \mathrm{wt} \%$, which was in good agreement with the value calculated based on the feed ratio of MAH and DVB (MAH:DVB feed ratio of $1.225 \mathrm{~g}: 1.1 \mathrm{~g}$; the 
elemental analysis data are shown in Supplementary Table S1). The anhydride-functionalized MSPP-A was hydrolyzed to convert the anhydride groups to carboxylic acid groups. The density of carboxylic acid groups in hydrolyzed MSPP-A was determined by titration and calculated to be approximately $9.2 \mathrm{mmol} \mathrm{g}^{-1}$, a little lower than the theoretical value calculated based on the monomer feed ratio $\left(9.8 \mathrm{mmol} \mathrm{g}^{-1}\right)$. This result was unexpected and demonstrated that, even though the MSPP was highly crosslinked, most of the carboxylic acid groups in the shell layer could behave as freely accessible reactive groups in a water-swollen state, which is essential for the quantitative adsorption of dye molecules. Remarkably, the density of the carboxylic acid groups is almost twice as high as that of previously reported carboxylic acid-functionalized mesoporous silica, ensuring the superior adsorption capacity of MSPP-A. ${ }^{26}$

In our present study, the carboxylate ions serve as the active binding sites for dye molecules. To facilitate the adsorption process, the carboxylate-functionalized MSPPs were used directly as the adsorbent for removal of the dye. As a proof of concept, we chose $b$-MB and methyl blue (acidic, $a-\mathrm{MB}$ ), which are routinely present in wastewaters, as the model dyes to characterize the adsorption performance of MSPP. A series of batch adsorption experiments were carried out with initial dye concentrations ranging from 50 to $3000 \mathrm{mgl}^{-1}$ at $\mathrm{pH}=7$ and 10 .

The equilibrium adsorption isotherms of the dye adsorption experiments are shown in Figure $4 \mathrm{a}$. It can be observed that the carboxylate-functionalized MSPP-A exhibited a remarkably high adsorption capacity for $b-\mathrm{MB}\left(1232 \mathrm{mgg}^{-1}\right.$ at $\left.\mathrm{pH}=7\right)$ and an extremely low adsorption capacity for $a-\mathrm{MB}\left(<1 \mathrm{mg} \mathrm{g}^{-1}\right)$, indicating that the carboxylate-functionalized MSPP-A is an efficient and selective adsorbent for basic dyes. Optical images were used to compare the adsorption performance of MSPP for $a-\mathrm{MB}$ and $b-\mathrm{MB}$, and it is obvious from Figure $4 \mathrm{~b}$ that the $b$-MB solution became clear and transparent within several minutes of treatment with the carboxylate-functionalized MSPP-A, whereas the $a$-MB solution remained unchanged.

Langmuir and Freundlich models were applied to analyze the isotherm data. Supplementary Figures S2a and b show the plot of $C_{\mathrm{e}} / q_{\mathrm{e}}$ against $C_{\mathrm{e}}$ for $b-\mathrm{MB}$, and the isotherm constants $\left(K_{\mathrm{L}}\right)$ and the maximum adsorption capacities $\left(Q_{\max }\right)$ are presented in Table $1 . C_{\mathrm{e}}$ is the equilibrium concentration of $b-\mathrm{MB}\left(\mathrm{mgl}^{-1}\right)$, and $q_{\mathrm{e}}$ is the adsorption capacity at equilibrium $\left(\mathrm{mgg}^{-1}\right)$. It is clear that the correlation coefficient was very high $(>0.999)$ and that the isotherms were linear over the whole concentration range, demonstrating that the $b-\mathrm{MB} /$ carboxylate-functionalized MSPP-A sorption data fit well with the Langmuir model. With increasing $\mathrm{pH}$ values, the adsorption capacity of carboxylate-functionalized MSPP-A dramatically increased owing to the higher content of $-\mathrm{COO}^{-}$ions at higher $\mathrm{pH}$ values. A remarkable maximum adsorption capacity of $1603 \mathrm{mgg}^{-1}$ was obtained at $\mathrm{pH}=10$, which is much larger than absorption capacities of $b$-MB reported for adsorbents such as carboxylic acid-functionalized mesoporous silica $\left(159 \mathrm{mg} \mathrm{g}^{-1}\right)^{26}$ and activated carbon (400-600 $\left.\mathrm{mg} \mathrm{g}^{-1}\right),{ }^{24}$ as well as reduced graphene oxide nanocomposite $\left(433 \mathrm{mgg}^{-1}\right){ }^{27}$ The plot of $\ln Q_{\mathrm{e}}$ versus $\ln C_{\mathrm{e}}$ for $b$-MB is given in Supplementary Figures S2c and d, and the Freundlich

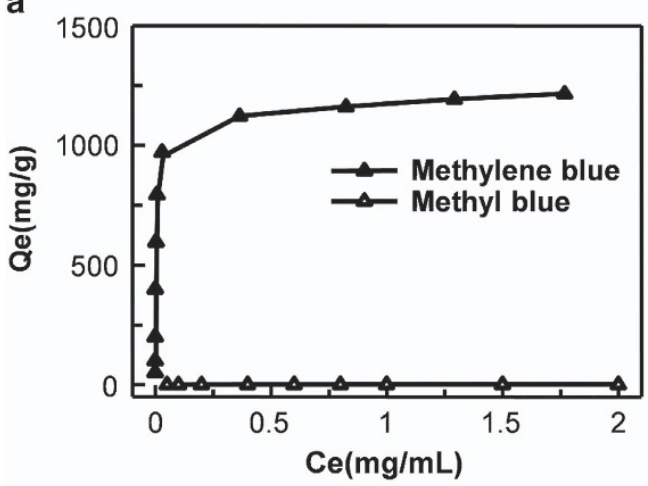

C

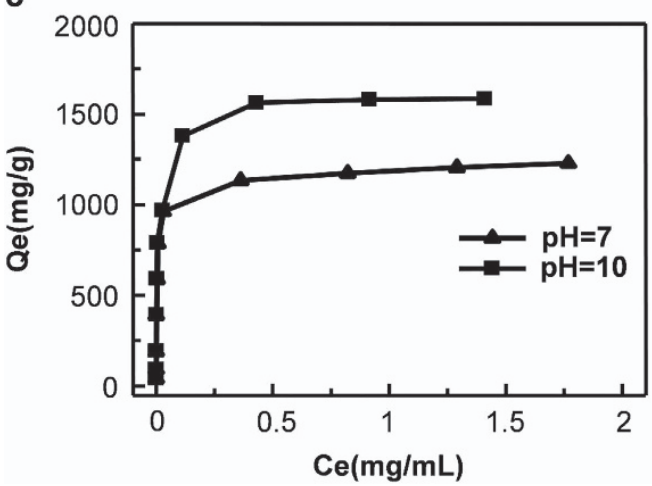

b

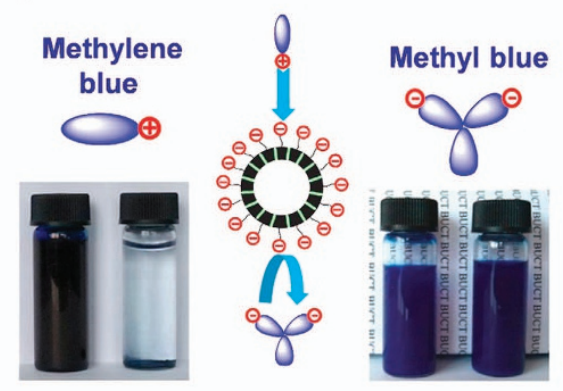

d



Figure 4 (a) Equilibrium adsorption isotherms of $b-\mathrm{MB}$ and $a-\mathrm{MB}$ onto the carboxylate-functionalized MSPP at pH 7.0. (b) Photographs of the dye solutions before and after treatment with the carboxylate-functionalized MSPPs. (c) Equilibrium adsorption isotherms of $b$-MB onto the carboxylate-functionalized MSPPs at different $\mathrm{pH}$ values. (d) The adsorption kinetics of $b$-MB onto the MSPPs at $\mathrm{pH}$ 7.0. $C_{\mathrm{e}}$ is the dye concentration in the aqueous phase at equilibrium, and $Q_{\mathrm{e}}$ is the adsorption capacity of the MSPPs. 
Table 1 Langmuir and Freundlich isotherm constants for $b$-MB dye on the hydrolyzed MSPPs at different $\mathrm{pH}$ values

\begin{tabular}{lcccrr}
\hline$p H$ & $\mathrm{~K}_{L}\left(/ \mathrm{mg}^{-1}\right)$ & $\mathrm{Q}_{\max }\left(m g^{-1}\right)$ & $\mathrm{R}^{2}$ & $\mathrm{~K}_{F}\left(m g g^{-1}\right) \times\left(/ m g^{-1}\right)^{1 / n}$ & $\mathrm{n}$ \\
\hline 7 & 0.0728 & 1232 & 0.9995 & 528.2 & 8.03 \\
10 & 0.0983 & 1603 & 0.9999 & 537.8 & 0.9047 \\
\hline
\end{tabular}

Abbreviations: $b-\mathrm{MB}$, basic methylene blue; MSPP, multilevel structured porous polymer particle.

constant $K_{\mathrm{F}}\left(\left(\mathrm{mg} \mathrm{g}^{-1}\right) \times\left(1 \mathrm{mg}^{-1}\right)^{1 / n}\right)$ and the heterogeneity factor $(1 / n)$ is presented in Table 1 . The results shown in Supplementary Figure S2 and Table 1 reveal that the fitting degree of the Freundlich model was slightly lower than that of the Langmuir model (correlation coefficient <0.95). In addition, the value of $n$ was in the range of 2-10 (8.03 and 5.93), indicating favorable adsorption activity of the carboxylate-functionalized MSPP to basic dyes.

The reason for this remarkable adsorption capacity can be easily understood based on the chemical property and the pore structure of the carboxylate-functionalized MSPP. The anhydride groups in the shell were converted to carboxylate groups after hydrolysis, and the 3D crosslinked networks could be partly swollen in aqueous medium, allowing for the carboxylate ions to effectively conjugate with dye molecules (similarly to that of hydrogels). More interestingly, after careful calculation, we found that the molar amount of $b-\mathrm{MB}$ adsorbed $\left(3.85 \mathrm{mmol} \mathrm{g}^{-1}, 1232 \mathrm{mg} \mathrm{g}^{-1}\right)$ was almost half of that of the carboxylate group density of MSPP $\left(8.06 \mathrm{mmol} \mathrm{g}^{-1}\right)$, indicating that each adsorbed $b$-MB molecule was bound to two carboxylate groups. Notably, because the hydrolysis of each anhydride group leads to two adjacent carboxylate groups, a $b$-MB molecule that is bound by one carboxylate group may not leave enough space for another $b$-MB molecule to bind owing to steric hindrance and electrostatic repulsion. Hence, it is reasonable to anticipate that the theoretical adsorption capacity of the carboxylate-functionalized MSPP-A is $4.03 \mathrm{mmol} \mathrm{g}^{-1}$ at $\mathrm{pH} 7.0$ or half the density of the carboxylate groups in MSPP. This theoretical adsorption capacity is in good agreement with the experimental results $\left(3.85 \mathrm{mmol} \mathrm{g}^{-1}\right)$, and this hypothesis is further confirmed by the adsorption capacity of crystal violet onto the carboxylate-functionalized MSPP-A $\left(q_{\mathrm{e}}=1550 \mathrm{mg} \mathrm{g}^{-1}, 3.80 \mathrm{mmol} \mathrm{g}^{-1}\right)$.

The adsorption capacity of $b-\mathrm{MB}$ onto the carboxylatefunctionalized MSPP-A could be further increased to $1603 \mathrm{mg} \mathrm{g}^{-1}$ at $\mathrm{pH}=10.0$, as shown in Figure $4 \mathrm{c}$. This extremely high adsorption capacity can be explained by the following two reasons: on one hand, the content of $\mathrm{COO}^{-}$ions became higher at higher $\mathrm{pH}$ values, consequently leading to higher adsorption capacity. On the other hand, the swelling degree of the crosslinked shell would increase accordingly with higher $\mathrm{pH}$ values owing to electrostatic repulsion, allowing more space for the diffusion and loading of $b$-MB molecules. As a result, the adsorption capacity increased dramatically by increasing the $\mathrm{pH}$ values up to 10.0.

As an advanced material, an adsorbent should not only have a high adsorption capacity but also present a rapid adsorption rate and excellent dye removal efficiency. The adsorption kinetics of $b$-MB onto MSPPs is shown in Figure 4d. Typically, the concentration of dyes in printing and dyeing wastewater is $<100-300 \mathrm{mgl}^{-1}$, which is an amount that can be efficiently removed by carboxylate-functionalized MSPP-A at dosages as low as $1 \mathrm{gl}^{-1}$. It can be clearly observed from Figure $4 \mathrm{~d}$ that the adsorption equilibrium of $b$-MB was attained in only $5 \mathrm{~min}$ for 600 and $800 \mathrm{mg} \mathrm{l}^{-1} b$-MB solution, whereas a longer time of $10 \mathrm{~min}$ was required for higher concentrations (that is, $1000 \mathrm{mg} \mathrm{l}^{-1}$ ), which is much shorter than equilibration times required for activated carbon $\left(6 \mathrm{~h}\right.$, for $100-300 \mathrm{mgl}^{-1}$; $>24 \mathrm{~h}$, for $400-500 \mathrm{mgl}^{-1}$; the dosage of adsorbent: $\left.1 \mathrm{gl}^{-1}\right){ }^{24}$ This impressive adsorption rate is mainly attributed to the unique hierarchical structure of the MSPP-A, which is both hollow and mesoporous. The hollow structure provides a high contact area, whereas the interconnected mesopores in the shell serve as a diffusion pathway, allowing for the rapid diffusion of the dye molecules into the interior of the MSPP. Furthermore, the strong affinity of carboxylate ions toward positively charged molecules further enhance the fast adsorption rate. In comparison, the adsorption rates of carboxylic acid-functionalized MSPP and non-hollow mesopore-free carboxylatefunctionalized DVB-MAH nanoparticles were much lower, as shown in Supplementary Figures S3a and b.

To provide greater insight into the effect of the pore structure on the adsorption properties, carboxylate-functionalized MSPP-D and MSPP-E having different mesoporous structures were prepared and evaluated as adsorbents for the removal of $b$-MB. The adsorption data of such mesoporous carboxylate-functionalized MSPPs were measured and compared in detail. As shown in Supplementary Figures S3c and d, the experimental results demonstrated that, although the adsorption capacities of these mesoporous carboxylate-functionalized MSPPs were almost identical, the adsorption rates were largely dependent on their surface area and pore structure. The mesoporous carboxylatefunctionalized MSPPs with lower surface area exhibited a much slower adsorption rate (based on $\mathrm{N}_{2}$ adsorption-desorption isotherms and pore size distribution, shown in Supplementary Figure S4; the surface area of MSPP-D and MSPP-E were 8.3 and $14.7 \mathrm{~m}^{2} \mathrm{~g}^{-1}$, respectively, and adsorption equilibrium was attained in $6-24 \mathrm{~h}$ for 200-400 $\mathrm{mg} \mathrm{l}^{-1}$ dye solution; the dosage of adsorbent: $1 \mathrm{gl}^{-1}$ ).

In addition to the remarkable adsorption capacity and rapid adsorption rate, the as-prepared carboxylate-functionalized MSPP-A also exhibited a high adsorption efficiency. Generally, the adsorption efficiency is strongly affected by the dye concentration and dosage of the adsorbing materials. Taking the high adsorption capacity into consideration, the dosage of the carboxylate-functionalized MSPP-A was set at $1 \mathrm{gl}^{-1}$, and the dye concentration was varied from 100 to $2000 \mathrm{mgl}^{-1}$. As shown in Figure 5a, the dye adsorption efficiency gradually decreased with increasing initial dye concentration, and the dye removal was $>99 \%$ even when the dye concentration was $800 \mathrm{mgl}^{-1}$ and when the residual dye concentration was as low as $7 \mathrm{mg} \mathrm{l}^{-1}$.

Additionally, the colloidal stability of the hollow polymer particle suspension was investigated at various MSPP concentrations and solution $\mathrm{pH}$ values. The slope of $n(d(\log A) / d(\log \lambda))$ as a function of MSPP concentration and solution $\mathrm{pH}$ is presented in Supplementary Figure S5. It can be concluded that the MSPP suspension appeared to have poor stability and that particle flocculation would take place over the whole concentration range. The complete precipitation of the MSPP suspension was achieved in 6-12 h owing to particle flocculation, which is beneficial for the separation and recovery of the adsorbent. To further characterize the stability and charge properties of the adsorbent, the zeta potential of the as-prepared carboxylatefunctionalized MSPPs was measured over the $\mathrm{pH}$ range of 1.0-10.0. As shown in Supplementary Figure S6, the isoelectric point of the carboxylate-functionalized MSPP was observed at a solution $\mathrm{pH}$ of 
a

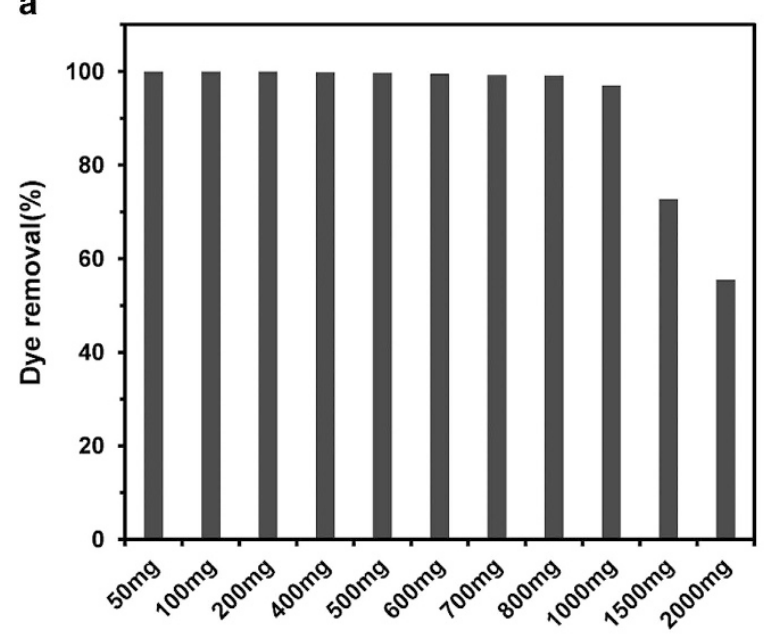

Dye concentration

C

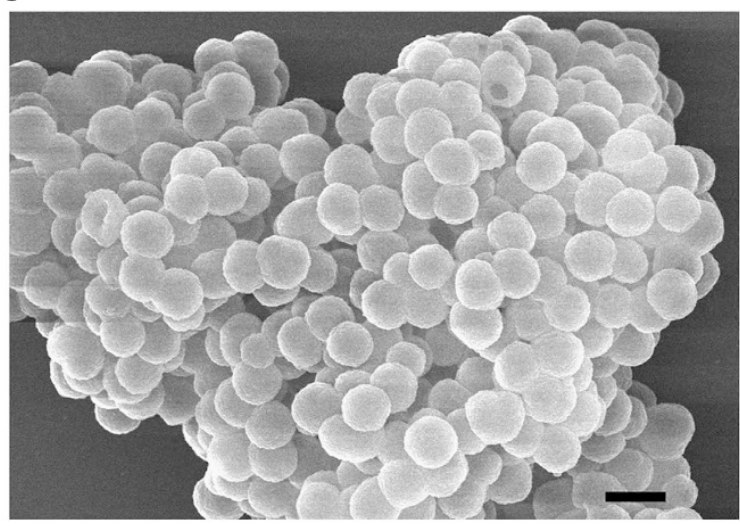

b

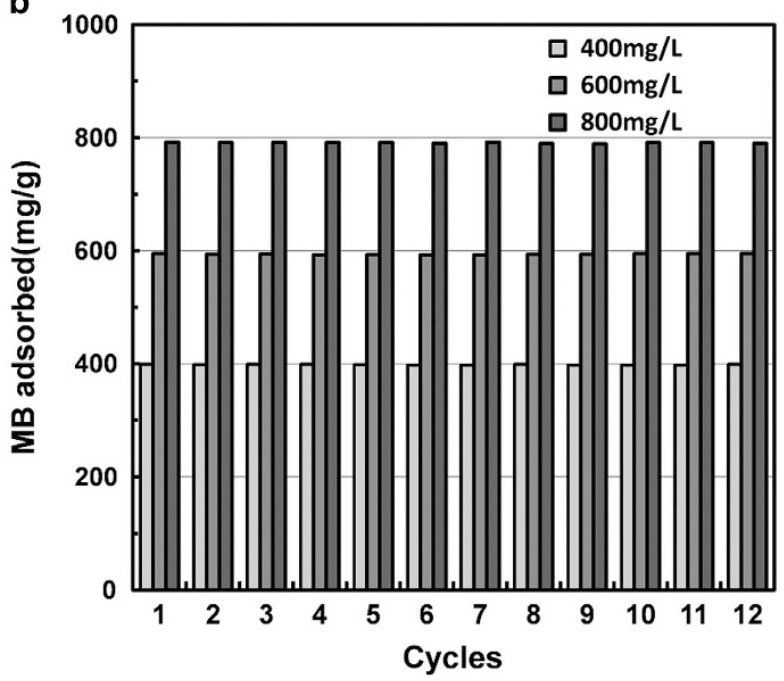

d

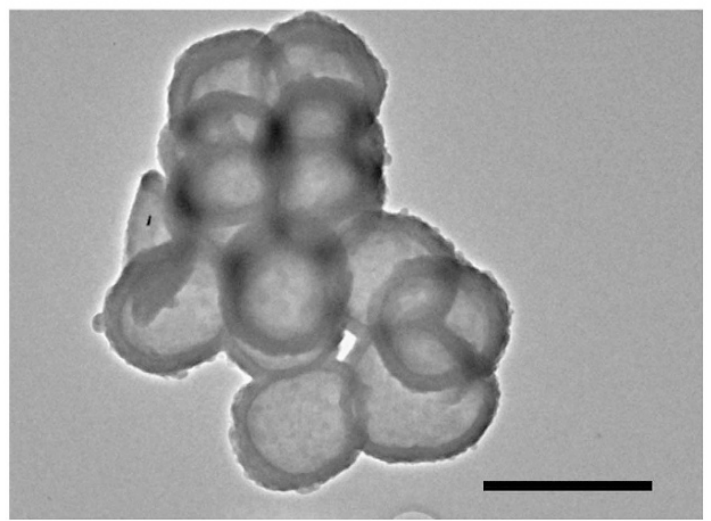

Figure 5 (a) Removal efficiency of $b$-MB by MSPPs for different concentrations of $b$-MB at pH 10.0. (b) The recyclability of MSPPs for the adsorption of $b$-MB. (c, d) SEM and TEM images of the MSPPs after 10 adsorption/desorption cycles. Scale bar: $1 \mu \mathrm{m}$.

approximately 3. Below this $\mathrm{pH}$ value, MSPP particles are positively charged, whereas the particles became negatively charged when the $\mathrm{pH}$ value was $>3$. Moreover, the zeta potential of MSPP particles decreased dramatically after dye adsorption, demonstrating that electrostatic interaction was the main mechanism for the dye adsorption. Owing to charge neutralization, the MSPP-A is easily precipitated from the suspension after the adsorption of $b-\mathrm{MB}$, and the thorough separation of the MSPP-A could be accomplished through simple centrifugation or filtration, as shown in Supplementary Figure S7.

From an application viewpoint, the regeneration and reusability of the adsorbent is just as important as its adsorption capacity and adsorption rate. As a high performance adsorbent, the carboxylatefunctionalized MSPP-A not only exhibited outstanding adsorption performance but also showed excellent desorption properties. The adsorbed $b$-MB could be readily desorbed in acidic ethanol (water/ethanol/HCl, $\mathrm{pH}=2-3$ ) to recover both the adsorbent and the adsorbed dye. The desorption experiment results are shown in Supplementary Figure S8, and an optical image of the regenerated adsorbent is presented in Supplementary Figure S9, which clearly show that the adsorbed $b$-MB was successfully desorbed; the calculated desorption efficiency reached $>95 \%$ after 3-4 desorption cycles in acidic ethanol. FT-IR spectra provided further evidence for the efficient removal of adsorbed dye molecules (as shown in
Supplementary Figure S10). This high desorption efficiency was attributed to the fact that the adsorption of $b-\mathrm{MB}$ onto the adsorbent was mainly due to electrostatic interactions between negatively charged $\mathrm{COO}^{-}$and the cationic dye molecules, which were very sensitive to the $\mathrm{pH}$ value of the solution. As a result, the absorbed $b$-MB can be desorbed efficiently under lower $\mathrm{pH}$ conditions during the desorption process.

The regenerated MSPP-A can be reused for the removal of $b-\mathrm{MB}$, and the adsorption efficiency was maintained over multiple adsorption-desorption cycles. Surprisingly, the dye removal efficiency decreased only slightly, to approximately $99 \%$, after 12 adsorptiondesorption cycles, even when the dye concentration was as high as $800 \mathrm{mg} \mathrm{l}^{-1}$ (Figure 5b), indicating that the as-prepared MSPP-A had good reusability. Figures $5 \mathrm{c}$ and $\mathrm{d}$ show the SEM and TEM images of the MSPP-A after 12 adsorption-desorption cycles, which show the high fidelity of the shell of the hollow particles, owing to its high degree of crosslinking.

We attribute the excellent adsorption property and recyclability of the MSPPs to their unique structure: MSPPs have high content of carboxylate groups in the crosslinked shell, giving it an extremely high adsorption capacity for basic dyes. In addition, the multilevel structure of our MSPPs, which include the presence of hollow voids, mesoporous channels and a 3D crosslinked network, are beneficial for 
fast diffusion of dye molecules and greatly increase the adsorption/ desorption rate. Furthermore, the high strength of the crosslinked shell facilitates the separation and outstanding recycling performance of the MSPPs. In view of all of the abovementioned advantages, the as-prepared MSPPs are an excellent, highly promising adsorbent material for dye removal from aqueous solution.

\section{CONCLUSION}

In summary, MSPPs with a multilevel structure, including submicron voids and interconnected mesopores within the crosslinked shell, have been specially designed and prepared through the precipitation copolymerization of DVB and MAH using in situ-formed PMS as a template, followed by the removal of the core via solvent etching. The content of anhydride groups in the crosslinked shell can reach up to $52.8 \mathrm{wt} \%$, which provides the MSPPs with the possibility of further modification with various functional groups for desired applications. As a proof of concept for practical applications, the MSPP particles were carboxylate functionalized and used as an advanced adsorbent for the removal of $b$-MB. Owing to the extremely high density of carboxylate groups $\left(8.06 \mathrm{mmolg}^{-1}\right)$ and the unique multilevel structure, the carboxylate-functionalized MSPP-A showed superior adsorption performance for the selective removal of $b$-MB $\left(1603 \mathrm{mg} \mathrm{g}^{-1}, 5.0 \mathrm{mmol} \mathrm{g}^{-1}\right)$. Meanwhile, the hollow structure and large mesopores $(15 \mathrm{~nm})$ in the rigid shell not only endow the adsorbent with fast adsorption/desorption kinetics but also facilitate its separation and regeneration. The adsorption equilibrium was attained in only $5 \mathrm{~min}$ for $800 \mathrm{mg} \mathrm{l}^{-1} b$-MB solution, and the adsorbed $b$-MB can be readily recovered with a high desorption efficiency (>95\%). The regenerated MSPPs can be reused following dye desorption, and the dye removal efficiency remained at approximately $99 \%$ even after 12 adsorption/desorption cycles. It is expected that, owing to their special multilevel structure and high density of functional groups, these MSPPs may have great potential in many fields, including as adsorbents for heavy metal ions, catalyst supports and matrices for the binding of sensitive materials.

\section{CONFLICT OF INTEREST}

The authors declare no conflict of interest.

\section{ACKNOWLEDGEMENTS}

This work was partly supported by 'the Fundamental Research Funds for the Central Universities' (No. ZY1405) and the National Natural Science Foundation of China (Grants 51033001, 51221002, 51273012 and 51403011).

1 Davis, M. E. Ordered porous materials for emerging applications. Nature 417, 813-821 (2002).

2 Li, J. R., Kuppler, R. J. \& Zhou, H. C. Selective gas adsorption and separation in metal-organic frameworks. Chem. Soc. Rev. 38, 1477-1504 (2009).

3 Zhang, Y. G. \& Riduan, S. N. Functional porous organic polymers for heterogeneous catalysis. Chem. Soc. Rev. 41, 2083-2094 (2012).

4 Kuznicki, S. M., Bell, V. A., Nair, S., Hillhouse, H. W., Jacubinas, R. M., Braunbarth, C. M., Toby, B. H. \& Tsapatsis, M. A titanosilicate molecular sieve with adjustable pores for size-selective adsorption of molecules. Nature 412, 720-724 (2001).

5 Vallet-Regi, M., Balas, F. \& Arcos, D. Mesoporous materials for drug delivery. Angew. Chem. Int. Ed. 46, 7548-7558 (2007).

6 Furukawa, H. \& Yaghi, O. M. Storage of hydrogen, methane, and carbon dioxide in highly porous covalent organic frameworks for clean energy applications. J. Am. Chem. Soc. 131, 8875-8883 (2009).

7 Liu, C., Li, F., Ma, L. P. \& Cheng, H. M. Advanced materials for energy storage. Adv. Mater. 22, E28-E62 (2010).

8 Sui, Z. Y., Meng, Y. N., Xiao, P. W., Zhao, Z. Q., Wei, Z. X. \& Han, B. H. Nitrogen-doped graphene aerogels as efficient supercapacitor electrodes and gas adsorbents. ACS Appl. Mater. Interfaces 7, 1431-1438 (2015).
9 Slater, A. G. \& Cooper, A. I. Function-led design of new porous materials. Science $\mathbf{3 4 8 ,}$ 988-998 (2015).

10 Soler-IIlia, G. J. \& Azzaronic, 0. Multifunctional hybrids by combining ordered mesoporous materials and macromolecular building blocks. Chem. Soc. Rev. 40, 1107-1150 (2011).

11 Thomas, A. Functional materials: from hard to soft porous frameworks. Angew. Chem. Int. Ed. 49, 8328-8344 (2010).

12 Nugent, P., Belmabkhout, Y., Burd, S. D., Cairns, A. J., Luebke, R., Forrest, K., Pham, T., Ma, S., Space, B., Wojtas, L., Eddaoudi, M. \& Zaworotko, M. J. Porous materials with optimal adsorption thermodynamics and kinetics for $\mathrm{CO} 2$ separation. Nature 495, 80-84 (2013).

13 Zhang, W., Liu, T. Y., Wu, H. H., Wu, P. \& He, M. Y. Direct synthesis of ordered imidazolyl-functionalized mesoporous polymers for efficient chemical fixation of $\mathrm{CO}_{2}$. Chem. Commun. 51, 682-684 (2015).

14 Makal, T. A., Li, J. R., Lu, W. G. \& Zhou, H. C. Methane storage in advanced porous materials. Chem. Soc. Rev. 41, 7761-7779 (2012).

$15 \mathrm{Wu}, \mathrm{Z}$. X. \& Zhao, D. Y. Ordered mesoporous materials as adsorbents. Chem. Commun. 47, 3332-3338 (2011).

16 Tan, M., Sum, Y. N., Ying, J. \& Zhang, Y. A mesoporous poly-melamine-formaldehyde polymer as a solid sorbent for toxic metal removal. Energy Environ. Sci. 6, 3254-3259 (2013).

17 Zhuang, X., Wan, Y., Feng, C. M., Shen, Y. \& Zhao, D. Y. Highly efficient adsorption of bulky dye molecules in wastewater on ordered mesoporous carbons. Chem. Mater. 21, 706-716 (2009).

18 Liang, C. Z., Sun, S. P., Li, F. Y., Ong, Y. K. \& Chung, T. S. Treatment of highly concentrated wastewater containing multiple synthetic dyes by a combined process of coagulation/flocculation and nanofiltration. J. Membr. Sci. 469, 306-315 (2014).

19 Zhang, X., Lin, M., Lin, X., Zhang, C., Wei, H., Zhang, H. \& Yang, B. Polypyrrole-enveloped $\mathrm{Pd}$ and $\mathrm{Fe} 3 \mathrm{O} 4$ nanoparticle binary hollow and bowl-like superstructures as recyclable catalysts for industrial wastewater treatment. ACS Appl. Mater. Interfaces 6, 450-458 (2014).

20 Chen, C., Ma, W. \& Zhao, J. Semiconductor-mediated photodegradation of pollutants under visible-light irradiation. Chem. Soc. Rev. 39, 4206-4219 (2010).

$21 \mathrm{Han}$, Y., Xu, Z. \& Gao, C. Ultrathin graphene nanofiltration membrane for water purification. Adv. Funct. Mater. 23, 3693-3700 (2013).

22 Sekar, S., Surianarayanan, M., Ranganathan, V., MacFarlane, D. R. \& Mandal, A. B. Choline-based ionic liquids-enhanced biodegradation of azo dyes. Environ. Sci. Technol. 46, 4902-4908 (2012).

23 Kyzas, G. Z., Fu, J. \& Matis, K. A. The change from past to future for adsorbent materials in treatment of dyeing wastewaters. Materials 6, 5131-5158 (2013).

24 Hameed, B. H., Din, A. T. M. \& Ahmad, A. L. Adsorption of methylene blue onto bamboo-based activated carbon: kinetics and equilibrium studies. J. Hazard. Mater. 141, 819-825 (2007).

25 Wang, S. \& Peng, Y. Natural zeolites as effective adsorbents in water and wastewater treatment. Chem. Eng. J. 156, 11-24 (2010).

26 Yan, Z., Tao, S. Y., Yin, J. X. \& Li, G. T. Mesoporous silicas functionalized with a high density of carboxylate groups as efficient absorbents for the removal of basic dyestuffs. J. Mater. Chem. 16, 2347-2353 (2006).

27 Gui, C. X., Wang, Q. Q., Hao, S. M., Qu, J., Huang, P. P., Cao, C. Y., Song, W. G. \& $\mathrm{Yu}, \mathrm{Z}$. Z. Sandwichlike magnesium silicate/reduced graphene oxide nanocomposite for enhanced $\mathrm{Pb} 2+$ and methylene blue adsorption. ACS Appl. Mater. Interfaces 6 , 14653-14659 (2014).

28 Pan, B. J., Pan, B. C., Zhang, W. M., Lv, L., Zhang, Q. X. \& Zheng, S. R. Development of polymeric and polymer-based hybrid adsorbents for pollutants removal from waters. Chem. Eng. J. 151, 19-29 (2009).

29 Chen, Y. N., He, M. F., Wang, C. Z. \& Wei, Y. M. A novel polyvinyltetrazole-grafted resin with high capacity for adsorption of $\mathrm{Pb}(\mathrm{II}), \mathrm{Cu}(\mathrm{II})$ and $\mathrm{Cr}(\mathrm{III})$ ions from aqueous solutions. J. Mater. Chem. A 2, 10444-10453 (2014).

30 Nayab, S., Farrukh, A., Oluz, Z., Tuncel, E., Tariq, S. R., Rahman, H. U., Kirchhoff, K., Duran, H. \& Yameen, B. Design and fabrication of branched polyamine functionalized mesoporous silica: an efficient absorbent for water remediation. ACS Appl. Mater. Interfaces 6, 4408-4417 (2014).

31 Yang, X. Y., Leonard, A., Lemaire, A., Tian, G. \& Su, B. L. Self-formation phenomenon to hierarchically structured porous materials: design, synthesis, formation mechanism and applications. Chem. Commun. 47, 2763-2786 (2011).

32 Shi, W. P., Tao, S. Y., Yu, Y. X., Wang, Y. C. \& Ma, W. High performance adsorbents based on hierarchically porous silica for purifying multicomponent wastewater. J. Mater. Chem. 21, 15567-15574 (2011).

33 Liang, Y. R., Li, Z. H., Fu, R. W. \& Wu, D. C. Nanoporous carbons with a 3D nanonetwork interconnected 2D ordered mesoporous structure for rapid mass transport. J. Mater. Chem. A 1, 3768-3773 (2013).

34 Ning, Y., Yang, Y., Wang, C. Y., Ngai, T. \& Tong, Z. Hierarchical porous polymeric microspheres as efficient adsorbents and catalyst scaffolds. Chem. Commun. 49, 8761-8763 (2013).

35 Meng, L. L., Zhang, X. F., Tang, Y. S., Su, K. H. \& Kong, J. Hierarchically porous silicon-carbon-nitrogen hybrid materials towards highly efficient and selective adsorption of organic dyes. Sci. Rep. 5, 7910 (2015).

36 Ray, S., Das, A. K., Banerjee, A., Ray, S., Das, A. K. \& Banerjee, A. pH-responsive, bolaamphiphile-based smart metallo-hydrogels as potential dye-adsorbing agents, water purifier, and vitamin B12 carrier. Chem. Mater. 19, 1633-1639 (2007). 
37 Gao, H. C., Sun, Y. M., Zhou, J. J., Xu, R. \& Duan, H. W. Mussel-inspired synthesis of polydopamine-functionalized graphene hydrogel as reusable adsorbents for water purification. ACS Appl. Mater. Interfaces 5, 425-432 (2013).

38 Deng, S. J., Xu, H. J., Jiang, X. S. \& Yin, J. Poly(vinyl alcohol) (PVA)-enhanced hybrid hydrogels of hyperbranched poly(ether amine) (hPEA) for selective adsorption and separation of dyes. Macromolecules 46, 2399-2406 (2013).

39 Xing, C. M. \& Yang, W. T. Facile method for the preparation of uniform, reactive maleic anhydride/vinyl acetate copolymer micro- and nanospheres. Macromol. Rapid Commun. 25, 1568-1574 (2004).

40 Deng, J. P., Yu, Y., Dun, S. \& Yang, W. T. Hollow polymer particles with nanoscale pores and reactive groups on their rigid shells: preparation and application as nanoreactors. J. Phys. Chem. B 114, 2593-2601 (2010). (c) (i) This work is licensed under a Creative Commons Attribution 4.0 International License. The images or other third party material in this article are included in the article's Creative Commons license, unless indicated otherwise in the credit line; if the material is not included under the Creative Commons license, users will need to obtain permission from the license holder to reproduce the material. To view a copy of this license, visit http:// creativecommons.org/licenses/by/4.0/

(C) The Author(s) 2016

Supplementary Information accompanies the paper on the NPG Asia Materials website (http://www.nature.com/am) 